\title{
Finite groups have even more conjugacy classes*
}

\author{
by
}

Thomas Michael Keller

Department of Mathematics

Texas State University

601 University Drive

San Marcos, TX 78666

USA

e-mail: keller@txstate.edu

2000 Mathematics Subject Classification: 20E45.

\footnotetext{
${ }^{*}$ This research was supported by the National Security Agency, Standard Grant No. 08G-206
} 
ABSTRACT. In his paper "Finite groups have many conjugacy classes" (J. London Math. Soc (2) 46 (1992), 239-249), L. Pyber proved the to date best general lower bounds for the number of conjugacy classes of a finite group in terms of the order of the group. In this paper we strengthen the main results in Pyber's paper.

\section{Introduction}

The subject of this paper are lower bounds for the number $k(G)$ of conjugacy classes of a finite group $G$ in terms of the group order. Over the years this fundamental and venerable problem has attracted the interest of a number of people, and progress has been slow, but steady, since Landau's well-known first observation in 1903 that $k(G)$ has to grow with $|G|$. In 1968, Erdös and Turán proved that $k(G)>\log _{2} \log _{2}|G|$, and since then this bound has been improved continuously for various classes of groups. We refer to E. A. Bertram's excellent survey [2] for more details on the history of this problem and the results obtained along the way. Suffice it to say here, that it is conjectured that a bound of the form

$$
(*) \quad k(G)>C \log |G|
$$

(for a constant $C$ ) is valid in general, and that there exist $p$-groups with $k(G)<(\log |G|)^{3}$. The currently best possible general bounds can be found in L. Pyber's paper Finite groups have many conjugacy classes [6], and the goal of this paper is to improve on the main results in [6]. We prove

Theorem A. There exists a (explicitly computable) constant $\epsilon_{1}>0$ such that every finite group $G$ with $|G| \geq 4$ satisfies

$$
k(G) \geq \epsilon_{1} \frac{\log _{2}|G|}{\left(\log _{2} \log _{2}|G|\right)^{7}}
$$

and if in addition $G$ is solvable, then even

$$
k(G) \geq \epsilon_{1} \frac{\log _{2}|G|}{\log _{2} \log _{2}|G|} .
$$

(See Corollary 3.2 and Corollary 3.3 below.)

Pyber obtained the same results with an exponent of 8 instead of 7 in the first part, and with a denominator of $\left(\log _{2} \log _{2}|G|\right)^{3}$ instead of $\log _{2} \log _{2}|G|$ in the second part. 
Two remarks are in order here. First, since our proof of Theorem A largely follows Pyber, the result for arbitrary groups depends on the Classification of Finite Simple Groups.

Second, as Pyber points out in his paper, a lower bound of the form $\epsilon \frac{\log |G|}{\log \log |G|}$ is the best possible that can be achieved with his approach. Thus our lower bound for solvable groups fully exhausts Pyber's approach, and any further progress will most likely have to be based on new stronger lower bounds for nilpotent groups than currently available.

Theorem A will be a consequence of the following improvement of Pyber's Theorem B in $[6]$ :

Theorem B. There is a (explicitly computable) constant $\beta>0$ such that for any solvable group with trivial Frattini subgroup we have

$$
k(G)>|G|^{\beta}
$$

(see Theorem 3.1 below.)

Theorems A and B will be proved in Section 3 of the paper. In Section 4 we will draw some consequences from Theorem B for solvable groups, thereby generalizing and improving some results in [2]. Perhaps the most interesting result of these is the following.

Theorem C. Let $G$ be a finite solvable group, and write c for the nilpotency class of the Frattini subgroup of $G$. Then

$$
k(G)>\left(\frac{1}{2} c|G|^{\frac{1}{c}}\right)^{\frac{\beta}{3}}
$$

where $\beta$ is the constant from Theorem $B$.

Notation: All groups in this paper are finite. If $G$ acts on a set $\Omega$, we write $n(G, \Omega)$ for the number of orbits of $G$ on $V$. If $V=V\left(n, q^{f}\right)$ is an $n$-dimensional vector space over $\operatorname{GF}\left(q^{f}\right)$ ( $q$ a prime power), then as in [5] we write $\Gamma\left(q^{f}\right)=\Gamma(V)=\left\{x \mapsto a x^{\sigma} \mid a \in\right.$ $\operatorname{GF}\left(q^{m}\right)^{x}, \sigma \in \operatorname{Gal}\left(\mathrm{GF}\left(q^{m}\right) / \mathrm{GF}(q)\right)$. Also log stand for $\log _{2}$. For any group $G$ we write $k(G)$ for the number of its conjugacy classes. If $G$ is a permutation group on a set $\Omega$, write $s(G)$ for the number of orbits of $G$ on the power set of $\Omega$. For any group, $F(G)$ and $\Phi(G)$ are its Fitting and Frattini subgroup, respectively. 


\section{Preliminaries}

In this section, for the convenience of the reader, we collect several known results from the literature that will be needed in the proof of the main results.

We will use a result of S. Seager [7], which we slightly reformulate here as in [4, Theorem 2.3] (note, however, that there is a typo in (ii) in the statement there).

2.1 Theorem. Let $G$ be a solvable group and $V$ a finite faithful irreducible $G$-module. Then one of the following holds:

(i) $|V| \leq\left(\frac{n(G, V)+1.43}{24^{\frac{1}{3}}}\right)^{c}$ for $c=36.435663$, so in particular, $n(G, V) \geq|V|^{\frac{1}{37}}$, or

(ii) for some integers $m, k$ with $k \leq 0.157 \log _{3}\left(\frac{n(G, V)+1.43}{2^{\frac{1}{3}}}\right) \leq \frac{1}{5} \log _{3} n(G, V)$ and a prime $p$ we have $|V|=p^{m k}$ and $G \lesssim \Gamma\left(p^{m}\right)$ ? $S_{k}$ in its natural action on $V(m, p)^{k}$.

Proof. See Theorem 1 in [7]. $\diamond$

2.2 Definition. We denote by $A$ the largest possible constant such that

$$
x^{\frac{1}{37}} \geq A \log x \quad \text { for all } x \geq 2 .
$$

Next we state a key lemma due to L. Pyber [6] and an immediate consequence which can also be found in [2].

2.3 Lemma. (a) Let $G$ be a group with $|G| \geq 4$. Suppose that $N \unlhd G$ is nilpotent and that

$$
k(G / N) \geq 2^{x(\log |G / N|)^{\frac{1}{t}}}
$$

for some constants $0<x \leq 1$ and $t \geq 1$. Then

$$
k(G) \geq \frac{x^{t} \log |G|}{2(\log \log |G|)^{t}} .
$$

(b) If $G$ is a group with $|G| \geq 4$ and $N \unlhd G$ is nilpotent such that $k(G / N) \geq|G / N|^{x}$ for some $0<x \leq 1$, then

$$
k(G) \geq \frac{x}{2} \frac{\log |G|}{\log \log |G|}
$$


Proof. For (a) see [6, Lemma 2.2], and (b) is the special case of (a) where $t=1$. $\diamond$

We also recall the following results:

2.4 Theorem. Let $0 \neq V$ be a faithful, completely reducible, finite $G$-module for a solvable group $G$. Then

$$
|G| \leq \frac{|V|^{\alpha}}{\lambda}
$$

where $\lambda=24^{\frac{1}{3}}=2 \cdot \sqrt[3]{3}$ and $9^{\alpha}=48 \lambda$, so that $2.24<\alpha<2.25$.

Proof. See [5, Theorem 3.5(a)]. $\diamond$

2.5 Lemma. There is an absolute constant $\alpha>0$ such that if $G$ is a solvable permutation group on a faithful set $\Omega$, then

$$
s(G) \geq|G|^{\alpha}
$$

Proof. This follows immediately from [6, Lemma 2.4]. (Note that our $\alpha$ here differs slightly from the $\alpha$ in Pyber's paper.)

We remark that the previous lemma is a special case of a more general (but elementary) result of Babai and Pyber [1] and that no explicit value of $\alpha$ is given in their work.

2.6 Lemma. Let $G \neq 1$ be a group and $N$ a normal subgroup of $G$. Suppose that $\alpha, \beta$ are real numbers with $0 \leq \alpha \leq 1$ and $0 \leq \beta \leq 1$ such that $k(N) \geq|N|^{\alpha}$ and $k(G / N) \geq|G / N|^{\beta}$. Then

$$
k(G)>|G|^{\frac{\alpha \beta}{1+\alpha+\beta}} \geq|G|^{\frac{\alpha \beta}{3}} .
$$

Proof. See [3, Lemma 3(i)]. $\diamond$ 


\section{The main results}

We first prove an improvement of a result of Pyber (see [6, Theorem B]). Pyber proved that if $G$ is solvable with $\Phi(G)=1$ and $|G| \geq 4$, then $k(G) \geq 2^{\beta \log |G| /(\log \log |G|)^{2}}$ for some universal constant $\beta>0$. Here we provide a polynomial lower bound in $|G|$ in the same situation.

3.1 Theorem. Let $G$ be a solvable group with $\Phi(G)=1$. Then

$$
k(G) \geq|G|^{\beta}
$$

for some universal constant $\beta>0$. Specifically, one can choose $\beta=\frac{\alpha}{1191(\alpha+1)+1}$ where $\alpha>0$ is as in Lemma 2.5.

Proof. We will use the well-known structural facts on solvable groups listed in [6, Lemmas 3.1 and 3.7] and also use some of the ideas from the proof of [6, Theorem B].

As is well-known, since $\Phi(G)=1, V=F(G)$ can be considered to be a faithful, completely reducible $H$-module of mixed characteristic, where $H$ is a complement of $F(G)$ in $G$. So we write $G=H V$ and write $V$ additively. More precisely, we can write $V=V_{1} \oplus \ldots \oplus V_{n}$ for some $n \in \mathbb{N}$ where each $V_{i}$ is an irreducible $\operatorname{GF}\left(p_{i}\right) G$-module for a prime $p_{i}(i=1, \ldots, n)$. (Note that the $p_{i}$ need not be mutually distinct.) Put $G_{i}=G / C_{G}\left(V_{i}\right) \cong H / C_{H}\left(V_{i}\right)$ for all $i$ and relabel the $V_{i}$ in such a way that $n\left(G_{i}, V_{i}\right) \geq\left|V_{i}\right|^{\frac{1}{37}}$ for all $i=1, \ldots, r$ for some $r \in\{0,1, \ldots, n\}$, and $n\left(G_{i}, V_{i}\right)<\left|V_{i}\right|^{\frac{1}{37}}$ for $i=r+1, \ldots, n$. Note that by Theorem 2.1, for $i=r+1, \ldots, n$, we know that $G_{i}$ has a normal subgroup $N_{i}$ of derived length at most 2 such that $G_{i} / N_{i}$ is isomorphic to a subgroup of $S_{k_{i}}$ for some $k_{i} \in \mathbb{N}$, and we can write

$$
(*) \quad V_{i}=V_{i 1} \oplus \ldots \oplus V_{i, k_{i}}
$$

with $N_{i}$-modules $V_{i j}$ which are permuted by $G_{i} / N_{i}$, so that

$$
N_{i}=\bigcap_{j=1}^{k_{i}} N_{G_{i}}\left(V_{i j}\right) \cong \bigcap_{j=1}^{k_{i}} N_{G}\left(V_{i j}\right) / C_{G}\left(V_{i}\right) .
$$

Now let $W_{1}=V_{1} \oplus \ldots \oplus V_{r}$ and $W_{2}=V_{r+1} \oplus \ldots \oplus V_{n}$. Clearly $W_{i} \unlhd G(i=1,2)$. Put

$$
H_{1}=C_{H}\left(W_{2}\right) \text { and } N=H_{1} W_{1} \unlhd G \text {. }
$$

Obviously $H_{1}$ acts faithfully on $W_{1}$. Next we put $H_{2}=H / H_{1}$ so that $H_{2}$ acts faithfully on $W_{2}$. Write $\mathrm{T}=\mathrm{H}_{2} W_{2}$ for the semidirect product of $H_{2}$ and $W_{2}$ with respect to that 
action and observe that $G / N \cong T$. (Note that if $r=0$, then $W_{1}=0, W_{2}=V, H_{1}=1$, $N=1$ and $T=G$; and if $r=n$, then $W_{1}=V, W_{2}=0, H_{1}=H, N=G$ and $T=1$.)

In view of Lemma 2.6, we now seek lower bounds for $k(T)$ and $k(N)$ separately. For $k(N)$, observe that clearly

$$
\begin{aligned}
k(N) & \geq n\left(H_{1}, W_{1}\right) \geq \prod_{i=1}^{r} n\left(H_{1}, V_{i}\right) \geq \prod_{i=1}^{r} n\left(G, V_{i}\right) \\
& =\prod_{i=1}^{r} n\left(G_{i}, V_{i}\right) \geq \prod_{i=1}^{r}\left|V_{i}\right|^{\frac{1}{37}}=\left|W_{1}\right|^{\frac{1}{37}}
\end{aligned}
$$

Moreover, by Theorem 2.4 we have $\left|H_{1}\right| \leq\left|W_{1}\right|^{3}$, and thus $|N| \leq\left|W_{1}\right|^{4}$, so altogether

$$
k(N) \geq\left|W_{1}\right|^{\frac{1}{37}} \geq\left(|N|^{\frac{1}{4}}\right)^{\frac{1}{37}}=|N|^{\frac{1}{148}}
$$

Next we study $k(T)$ and obtain a lower bound similarly as in the proof of [6, Theorem B]. If $i \in\{r+1, \ldots, n\}$, then we observe that $G_{i} \cong H / C_{H}\left(V_{i}\right)$ and thus let $M_{i} \leq H$ be the inverse image of $N_{i}$ in $H$. Hence $M_{i} / C_{H}\left(V_{i}\right)=N_{i}$ and hence

$$
M_{i}=\bigcap_{j=1}^{k_{i}} N_{H}\left(V_{i j}\right) .
$$

Put

$$
\Omega=\left\{V_{i j} \mid i=r+1, \ldots, n ; j=1, \ldots, k_{i}\right\} .
$$

Clearly $H$ acts on $\Omega$, and if we let

$$
K=\bigcap_{i=r+1}^{n} \bigcap_{j=1}^{k_{i}} N_{H}\left(V_{i j}\right)=\bigcap_{i=r+1}^{n} M_{i}
$$

be the kernel of this action, then $H / K \lesssim \operatorname{Sym}(\Omega)$. We also claim that

$$
\text { (2) } K / H_{1} \lesssim X_{i=r+1}^{n} N_{i} \text {. }
$$

To see this, observe that clearly $1=\bigcap_{i=1}^{n} C_{H / H_{1}}\left(V_{i}\right)$. Therefore, if for $U \leq H$ we write $\bar{U}=U H_{1} / H_{1}$, then

$$
\bar{K} \cong \bar{K} / \bigcap_{i=r+1}^{n}\left(\bar{K} \cap C_{\bar{H}}\left(V_{i}\right)\right)
$$




$$
\begin{aligned}
& \leqq X_{i=r+1}^{n} \bar{K} /\left(\bar{K} \cap C_{\bar{H}}\left(V_{i}\right)\right) \\
& \cong X_{i=r+1}^{n}\left(\bar{K} C_{\bar{H}}\left(V_{i}\right)\right) / C_{\bar{H}}\left(V_{i}\right) \\
& \leq X_{i=r+1}^{n} \overline{M_{i}} / C_{\bar{H}}\left(V_{i}\right) \\
& =X_{i=r+1}^{n}\left(M_{i} / H_{1}\right) /\left(C_{H}\left(V_{i}\right) / H_{1}\right) \\
& \cong X_{i=r+1}^{n} M_{i} / C_{H}\left(V_{i}\right) \\
& =X_{i=r+1}^{n} N_{i}
\end{aligned}
$$

which proves the claim.

Now as $\operatorname{dl}\left(N_{i}\right) \leq 2$ for $i=r+1, \ldots, n$, from (2) we see that for the semidirect product $\bar{K} W_{2} \leq T$ we have

$$
\mathrm{dl}\left(\bar{K} W_{2}\right) \leq \mathrm{dl}(\bar{K})+\operatorname{dl}\left(W_{2}\right) \leq 2+1=3 .
$$

Now a result of Bertram [3, Theorem 1] yields

$$
k\left(\bar{K} W_{2}\right) \geq\left|\bar{K} W_{2}\right|^{\frac{1}{7}}
$$

On the other hand,

$$
S:=T /\left(\bar{K} W_{2}\right) \cong \bar{H} / \bar{K} \cong H / K \leqq \operatorname{Sym}(\Omega)
$$

and thus by Lemma 2.5 we have

$$
s(S) \geq|S|^{\alpha}
$$

with the $\alpha$ as in Lemma 2.5. Now if two elements $x_{k} \in W_{2}(k=1,2)$ are conjugate in $G$, then they are conjugate in $T$, and if we write

$$
v_{k}=\sum_{i=r+1}^{n} \sum_{j=1}^{k_{i}} \alpha_{k i j} v_{k i j}
$$

for suitable $v_{k i j} \in V_{i j}$ and $\alpha_{k i j}$ in the field belonging to $V_{i j}(k=1,2)$, and $t \in T$ is such that $v_{1}^{t}=v_{2}$, then clearly for the subsets $\Omega_{k}=\left\{V_{i j} \mid \alpha_{k i j} v_{k i j} \neq 0\right\}$ of $\Omega(k=1,2)$ we have that $\Omega_{1}^{t \bar{K} W_{2}}=\Omega_{2}$. Therefore

$$
k(T) \geq n\left(\bar{H}, W_{2}\right) \geq s(S) \geq|S|^{\alpha} .
$$

Now with (3) and [6, Lemma 2.1(ii)] we also get

$$
k(T) \geq \frac{\left|\bar{K} W_{2}\right|^{\frac{1}{7}}}{|S|}
$$

and putting (4) and (5) together gives us

$$
|T|=|S|\left|\bar{K} W_{2}\right| \leq|S||S|^{7} k(T)^{7}=|S|^{8} k(T)^{7} \leq k(T)^{\frac{8}{\alpha}} k(T)^{7}=k(T)^{\frac{8}{\alpha}+7} .
$$


Therefore

$$
k(G / N)=k(T) \geq|T|^{\frac{1}{\alpha}+7}=|T|^{\frac{\alpha}{8+7 \alpha}} \quad(6) .
$$

Finally, using (1), (6), and Lemma 2.6, we obtain

$$
k(G) \geq|G|^{\beta} \text { with } \beta=\frac{\alpha}{1148+1036 \alpha} /\left(\frac{149}{148}+\frac{\alpha}{8+7 \alpha}\right)=\frac{\alpha}{1191(\alpha+1)+1},
$$

as desired.

Our first application are improvements on the strongest general lower bounds for $k(G)$ in terms of $|G|$ for solvable and arbitrary finite groups, as obtained by Pyber in [6]. As to solvable groups, Pyber's arguments show that

$$
k(G) \geq \beta_{1} \frac{\log |G|}{(\log \log |G|)^{3}}
$$

for a suitable (universal) constant $\beta_{1}$ (see [2, Corollary 2.1]). Here we improve this as follows.

3.2 Corollary. Let $G$ be a solvable group with $|G| \geq 4$, and let $\beta$ be as in Theorem 3.1. Then

$$
k(G) \geq \frac{\beta}{2} \frac{\log |G|}{\log \log |G|} .
$$

Proof. Let $N=\Phi(G)$. By Theorem 3.1 we know that $k(G / N) \geq|G / N|^{\beta}$, so by Lemma 2.3(b) we get the desired conclusion.

For arbitrary groups, the best possible bound to date is Pyber's result that there exists an $\epsilon>0$ such that

$$
k(G) \geq \epsilon \frac{\log |G|}{(\log \log |G|)^{8}} .
$$

Using Theorem 2.1 in Pyber's proof, we can get the following slight improvement:

3.3 Corollary. There exists a constant $\epsilon_{1}>0$ such that if $G$ is a group with $|G| \geq 4$, then

$$
k(G) \geq \epsilon_{1} \frac{\log |G|}{(\log \log |G|)^{7}} .
$$


Proof. Let $O=O_{\infty}(G)$ be the largest normal solvable subgroup of $G$, so clearly $O_{\infty}(G / O)=1$. Let $\Phi=\Phi(O)$, and let $x$ and $y$ denote the orders of the factor groups $O / \Phi$ and $G / O$, respectively. Then $\Phi(O / \Phi)=1$, and thus by Theorem 3.1 we have $k(O / \Phi) \geq x^{\beta}$, where $\beta$ is as in Theorem 3.1. If follows that

$$
k(G / \Phi) \geq \frac{k(O / \Phi)}{|G / O|} \geq \frac{x^{\beta}}{y} .
$$

Thus

(1) $\log k(G / \Phi) \geq \beta \log x-\log y=\beta \log (x y)-(1+\beta) \log y=\beta \log |G / \Phi|-(1+\beta) \log y$.

Now by [6, Lemma 4.7] we have

$$
\text { (2) } \log k(G / \Phi) \geq \log k(G / O) \geq \delta(\log y)^{\frac{1}{7}} .
$$

Combining (1) and (2) therefore yields

$$
\begin{aligned}
\beta \log |G / \Phi| & \leq \log k(G / \Phi)+(1+\beta) \log y \\
& \leq \log k(G / \Phi)+(1+\beta)\left(\frac{\log k(G / \Phi)}{\delta}\right)^{7} .
\end{aligned}
$$

This easily implies that

$$
\log k(G / \Phi) \geq \gamma(\log |G / \Phi|)^{\frac{1}{7}}
$$

for some $0<\gamma \leq 1$. Thus

$$
k(G / \Phi) \geq 2^{\gamma(\log |G / \Phi|)^{\frac{1}{7}}},
$$

and as $\Phi$ is nilpotent, we may apply Lemma 2.3(a) which yields the assertion.

\section{More applications for solvable groups}

In this section we significantly strengthen some results of Bertram [2] on solvable groups. In [2, Proposition 2.3] it is shown that if $G$ is solvable, $\Phi(G)$ is abelian and $|G|$ is sufficiently large (depending only on $t$ ), then $k(G)>(\log |G|)^{t}$. We now strengthen the lower bound and generalize the hypothesis to allow $\Phi(G)$ to be of a fixed (arbitrary) nilpotency class. What we get somewhat resembles Sherman's bound $k(P) \geq c|P|^{\frac{1}{c}}-c+1$ for nilpotent groups of nilpotency class $c$ (see [8]). 
4.1 Theorem. Let $G$ be a solvable group, and suppose $\Phi(G)$ is of nilpotency class $c$. Then

$$
\log k(G)>\frac{\beta\left(\frac{1}{c}+\frac{\log (c / 2)}{\log |\Phi|}\right)}{1+\beta+\frac{1}{c}+\frac{\log (c / 2)}{\log |\Phi|}} \log |G|,
$$

where $\beta$ is as in Theorem 3.1. In particular,

$$
k(G)>\left(\frac{1}{2} c|G|^{\frac{1}{2}}\right)^{\frac{\beta}{3}} .
$$

Moreover, if $\Phi(G)$ is abelian, then

$$
k(G)>|G|^{\frac{\beta}{2+\beta}} \geq|G|^{\frac{\beta}{3}}
$$

Proof. Write $\Phi=\Phi(G)$. By Theorem 3.1 we have $k(G / \Phi) \geq|G / \Phi|^{\beta}$. Also, by Sherman's result [8] we have $k(\Phi) \geq c|\Phi|^{\frac{1}{c}}-c+1$. As $|\Phi|^{\frac{1}{c}} \geq 2$, it is easy to see that this implies that $k(\Phi) \geq \frac{c}{2}|\Phi|^{\frac{1}{c}}=|\Phi|^{\frac{1}{c}+\frac{\log \frac{c}{2}}{\log |\Phi|}}$. Thus by Lemma 2.6 we conclude that

$$
\log k(G)>\frac{\beta\left(\frac{1}{c}+\frac{\log (c / 2)}{\log |\Phi|}\right)}{1+\beta+\frac{1}{c}+\frac{\log (c / 2)}{\log |\Phi|}} \log |G| \geq \frac{\frac{\beta}{c}+\frac{\beta \log (c / 2)}{\log |\Phi|}}{3} \log |G| .
$$

Hence

$$
k(G) \geq|G|^{\frac{\beta}{3 c}} \cdot 2^{\frac{\beta}{3} \log (c / 2)}=|G|^{\frac{\beta}{3 c}}\left(\frac{c}{2}\right)^{\frac{\beta}{3}}=\left(\frac{1}{2} c|G|^{\frac{1}{c}}\right)^{\frac{\beta}{3}},
$$

as claimed. Finally, if $\Phi$ is abelian, then $k(\Phi)=|\Phi|$, and then Lemma 2.6 yields

$$
k(G)>|G|^{\frac{\beta}{2+\beta}} \geq|G|^{\frac{\beta}{3}} \text {, andtheproofiscomplete. }
$$

$\diamond$

Our next goal is to strengthen [2, Corollary 2.3].

4.2 Corollary. Let $G$ be solvable, and write $F=F(G)$ for the Fitting subgroup of $G$. Let $\beta$ be as in Theorem 3.1.

(a) Let $0<\alpha \leq 1$. If $\left|F^{\prime}\right| \leq|G|^{1-\frac{\alpha(2+\beta)}{\beta}}$, then $k(G)>|G|^{\alpha}$. 
(b) Let $t>0$. If $\left|F^{\prime}\right| \leq \frac{|G|}{(\log |G|)^{t\left(1+\frac{2}{\beta}\right)}}$, then $k(G)>(\log |G|)^{t}$.

Proof. As in the proof of [2, Corollary 2.3] we see that

$$
\Phi\left(G / F^{\prime}\right)=\Phi(G) / F^{\prime}<F / F^{\prime}
$$

and thus $\Phi\left(G / F^{\prime}\right)$ is abelian. By Theorem 4.1 we conclude that $k(G) \geq k\left(G / F^{\prime}\right)>$ $\left|G / F^{\prime}\right|^{\frac{\beta}{2+\beta}}$. Now $\left|G / F^{\prime}\right|^{\frac{\beta}{2+\beta}} \geq|G|^{\alpha}$ if and only if $\left|F^{\prime}\right| \leq|G|^{1-\frac{\alpha(2+\beta)}{\beta}}$, as can easily be checked. This is (a).

Likewise, $\left|G / F^{\prime}\right|^{\frac{\beta}{2+\beta}} \geq(\log |G|)^{t}$ if and only if $\left|F^{\prime}\right|$ satisfies the hypothesis in (b), and so we are done.

We finally improve [2, Corollary 2.4].

4.3 Corollary. Let $G$ be a solvable Frobenius group with Frobenius kernel $N$. If $N$ is abelian, then

$$
k(G)>|G|^{\frac{\beta}{2+\beta}}
$$

where $\beta$ is as in Theorem 3.1.

Proof. As in the proof of [2, Corollary 2.4] we note that $N=F(G)$, and so we are done by Theorem 4.1 .

As already remarked in [2], note that if $G$ is a Frobenius group with Frobenius kernel $N$ such that $|G / N|$ is even, then $N$ necessarily is abelian and Corollary 4.3 can be applied.

\section{References}

[1] L. Babai, L. Pyber, Permutation groups without exponentially many orbits on the power set, J. Combin. Theory Ser. A 66 (1994), 160-168.

[2] E. A. Bertram, Lower bounds for the number of conjugacy classes in finite groups, Ischia Group Theory 2004, 95-117, Contemp. Math. 402, AMS, Providence, RI, 2006.

[3] E. A. Bertram, Lower bounds for the number of conjugacy classes in finite solvable groups, Israel J. Math. 75 (1991), 243-255.

[4] T. M. Keller, Orbits in finite group actions, Groups St. Andrews 2001 in Oxford, LMS Lecture Note Series 305, Cambridge University Press, 2003. 
[5] O. Manz and T. R. Wolf, Representations of solvable groups, London Math. Soc. Lecture Notes Series 185, Cambridge University Press, 1993.

[6] L. Pyber, Finite groups have many conjugacy classes, J. London Math. Soc. (2) 46 (1992), 239-249.

[7] S. M. Seager, A bound on the rank of primitive solvable permutation groups, J. Algebra 116 (1988), 342-394.

[8] G. J. Sherman, A lower bound for the number of conjugacy classes of a finite nilpotent group, Pacific J. Math 80 (1979), 253-254. 